\title{
Feedback control baseline for GTC adaptive optics with NGS
}

M. Núñez Cagigal ${ }^{* a}$, L. F. Rodríguez Ramos ${ }^{\mathrm{a}}$, O. Tubio Araujo ${ }^{\mathrm{a}}$, Jose Marco de la Rosa ${ }^{\mathrm{a}}$, Alastair Basden ${ }^{\mathrm{b}}$, Iciar Montilla ${ }^{a}$, Josefina Rosich Minguell a, Roberto López López a , Marta Puga Antolín a , Marcos Reyes García-Talavera a Jesús Patrón Recio ${ }^{a}$

anstituto de Astrofísica de Canarias (IAC), La Laguna, Spain. *migueln@iac.es

${ }^{b}$ Durham University, England.

\begin{abstract}
Gran Telescopio Canarias Adaptive Optics (GTCAO) with NGS is a single-conjugated post-focal system with a Shack Hartmann wavefront sensor at visible wavelength, one Deformable Mirror (DM) and the telescope secondary mirror that is used to correct partially the tip-tilt. GTCAO feeds the scientific instrument FRIDA, which is an imager and integral field spectrograph with an image slicer.

Bandwidth error has been calculated creating models for tip-tilt and high order optical modes. The error functions has been applied to representative atmospheric conditions that has been chosen based on sky characterization data from "Observatorio del Roque los Muchachos" such as coherence time or the guide star flux. Three different strategies are analysed for tip-tilt correction and one of them is proposed to be implemented.

The main difference between the implementation in laboratory and the commissioning in telescope is the absence of the secondary mirror in the laboratory, an important element in the control loop that will off-load the DM, correcting tip-tilt perturbations at low temporal frequencies.
\end{abstract}

Keywords: Adaptive optics, servo control, GTC

\section{INTRODUCTION}

The GTCAO [1][2][3][4] is a single conjugated post focal AO system placed in the GTC Nasmyth platform over a static optical table. GTCAO follows the classic design of an AO system with the use of two identical off-axis parabolas, maintaining the effective focal distance of the telescope. It has been designed initially for Natural Guide Star (NGS) and in a later project phase adapted to one Laser Guide Star (LGS). After the AO system, the FRIDA[5] science instrument will be installed, which is a near infrared spectrograph that will offer broad and narrow band imaging and integral field spectroscopy capabilities with low (1000), intermediate (4500) and high (30000) spectral resolutions to operate in the wavelength range $0.9-2.5 \mu \mathrm{m}$. Main high level specifications are in table 1 .

\begin{tabular}{|c|c|}
\hline Wavelength & 1.0-2.5 micron, with a goal of $0.8-5$ micron \\
\hline Strehl Ratio & $\begin{array}{l}\mathrm{SR}>=0.65 \text { at } 2.2 \text { micron for a bright } \mathrm{NGS} \text { on axis } \\
\mathrm{SR}>=0.1 \text { at } 2.2 \text { micron for a faint } \mathrm{NGS}\left(\mathrm{m}_{\mathrm{R}}=14.5\right)\end{array}$ \\
\hline Range of operation & $\begin{array}{l}\text { Seeing better than } 1.5 \operatorname{arcsec} \text { FWHM at } 500 \mathrm{~nm} \\
\text { Zenith angles } 0^{\circ}-60^{\circ}\end{array}$ \\
\hline Field of View (FoV) & $\begin{array}{l}1.5 \text { arcmin available to the science instrument } \\
2.0 \text { arcmin accessible for wavefront sensing }\end{array}$ \\
\hline Observation time & At least $1 \mathrm{~h}$ exposure time on the science instrument \\
\hline Dithering & Offsets of 0.25 arcsec (goal 1.0 arcsec) without interrupting operation \\
\hline Nodding & $\begin{array}{l}\text { Ability to keep the loop closed while nodding the telescope at } 1 \text { arcsec per } \\
\text { second (TBC) }\end{array}$ \\
\hline Throughput & $\begin{array}{l}\text { Throughput of wavefront corrector shall be at least } 70 \% \text { in the wavelength range } \\
\text { from } 1.0 \text { to } 2.5 \text { micron with a goal of } 70 \% \text { in the range } 0.8 \text { to } 5 \text { micron }\end{array}$ \\
\hline Emissivity & $<20 \%$ at 3.8 micron \\
\hline Ghost images & $\begin{array}{l}\text { Defocused ghosts: }<1 \mathrm{e}-5 \text { (except dichroic } 1 \mathrm{e}-4) \\
\text { Focused ghosts: }<1 \mathrm{e}-3 \text { and located within } 0.2 \text { arcsec) }\end{array}$ \\
\hline Upgrades & First upgrade for the use of a single Laser Guide Star \\
\hline
\end{tabular}

Table 1. GTCAO main high level requirements. 


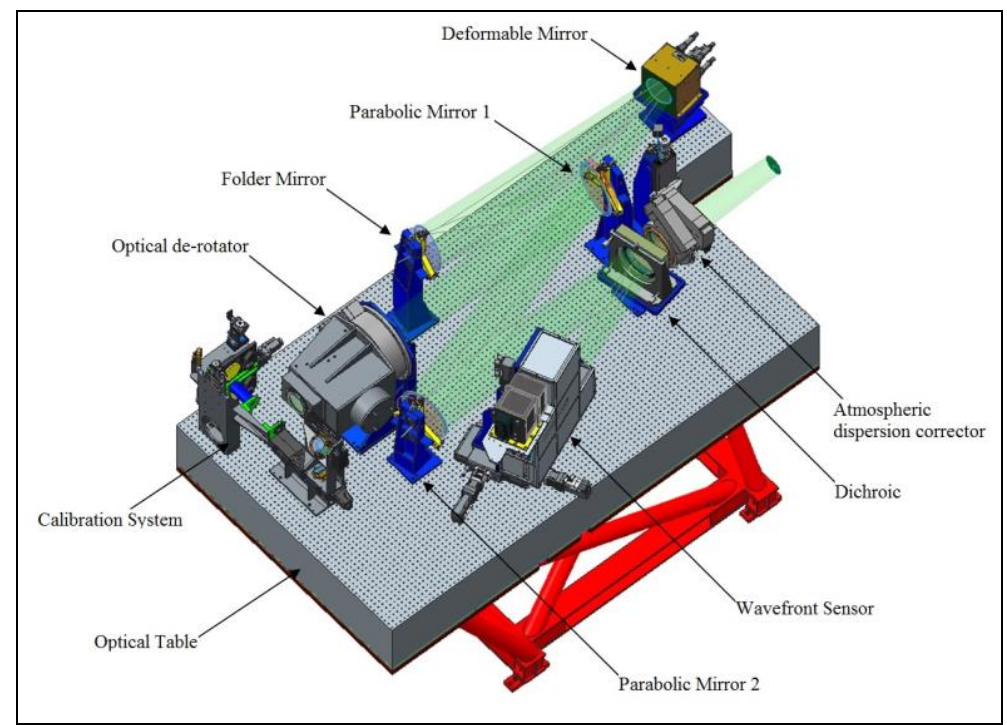

Figure 1. GTCAO optical bench with all subsystems and cable conduits.

The Deformable Mirror (DM) has 373 actuators, some of them are out of the pupil projection but they are used to shape the mirror in the pupil edges. The shack-hartman Wave Front Sensor (WFS) has 20x20 subapertures of which about 356 may be illuminated including certain misalignment as shown in Figure 2.

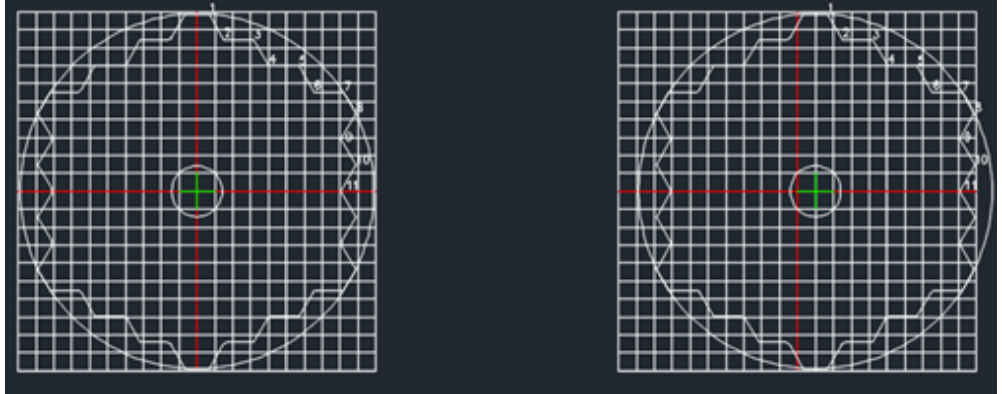

Figure 2. Projection of telescope entrance pupil on WFS micro-lens array. Left: nominal position. Right: one sub aperture displacement.

GTC M1 is a 10.4m segmented mirror with 36 hexagonal segments, including each one edge sensors and 3 position actuators. M1 has a central obscuration of about $5.6 \%$ of the whole surface. M1 position can be defined optically as global focus, global coma and 36x piston, tip and tilt, making a 110 elements vector. Additionally to stacking and phasing, M1 will off-load DM.

M2 is a monolithic lightweight convex hyperbolic mirror that defines the telescope pupil and it can produce movements in $\mathrm{x}, \mathrm{y}$, focus, tip and tilt.

Tip-tilt measured in the WFS will be corrected by both M2 and the DM. M2 is in charge of correcting the temporally slow tip-tilt and also of off-loading the DM from accumulated tip-tilt. The DM is in charge of correcting temporally fast tip-tilt and other high order optical modes.

\section{WAVEFRONT DISTURBANCE SOURCES}

In this section a number of different atmospheric conditions are selected, providing, as a whole, a reasonable model of the expected turbulence at GTC site. Wavefront disturbance models are presented considering separately the spatial high order and spatial low order modes. 


\subsection{Atmosphere conditions}

Using data provided by IAC High Spatial Resolution Group (HSRG) three different conditions have been chosen which represent different percentiles of nights at Observatorio del Roque de los Muchachos (ORM). The measured data were $\mathrm{Cn}^{2}$ profiles using a SCIDAR instrument installed in the Jacobus Kaptein Telescope, and wind at high altitudes in the same dates and times, measured by meteorological balloons launched from Güimar (Tenerife). The acquired data was processed by HSRG using methodology described in [6]. The results are 140 sets of values including average velocity of the turbulence $(\mathrm{v} 0)$, Fried parameter $(\mathrm{r} 0)$, atmospheric coherence time $\left(\tau_{0}\right)$ and date. With that data, three atmospheric conditions are selected:

1. GTCAO Standard Scenario:

a. Equivalent wind $=10 \mathrm{~m} / \mathrm{s}$.

b. $\mathrm{r} 0=0.2 \mathrm{~m}$.

c. Atmospheric coherence time $=6.3 \mathrm{~ms}$.

2. Median atmosphere:

a. Equivalent wind $=10 \mathrm{~m} / \mathrm{s}$.

b. $\mathrm{r} 0=0.156 \mathrm{~m}$.

c. Atmospheric coherence time $=4.9 \mathrm{~ms}$.

3. Adverse atmosphere:

a. Equivalent wind $=15 \mathrm{~m} / \mathrm{s}$.

b. $\mathrm{r} 0=0.067 \mathrm{~m}$. This is the limit of the GTCAO specification seeing $=1.5$ arcsec .

c. Atmospheric coherence time $=1.4 \mathrm{~ms}$.

\subsection{Spatial low order. Tip/tilt}

Tip-tilt atmospheric wavefront aberrations produce a movement of the whole image in the focal plane, but there are other sources that can produce whole image movement in the focal plane. All of them act simultaneously over the image received by GTCAO wavefront sensor and by AO instrument (FRIDA), accumulating their independent effects. The main contributors are:

1. Atmospheric-induced tip-tilt.

2. Windshake-induced tip-tilt

3. Other residual tip-tilt error produced by telescope as the telescope tracking error which can be corrected based on AO WFS

Equation 1, obtained from [7], is used to model the tip tilt Power Spectrum Density (PSD) obtaining Figure 3 for 2 different atmospheres: GTCAO standard seeing scenario and adverse atmosphere defined previously. The adverse atmosphere produces a higher tip-tilt disturbance at all the frequencies. The windshake does not change with atmospheric conditions, it has a RMS amplitude of $0.1 \mathrm{arcsec}$ and its percentage of influence is higher when the atmosphere has better conditions. The 0.1 arcsec amplitude is an estimation extracted from [6][7]. It has been proposed to carry out specific measurements in GTC telescope to better quantify the effect of wind over tip tilt. 


$$
\begin{aligned}
& P S D_{T T}(f)=\left\{\begin{array}{lll}
0.804 D^{-1 / 3} v_{-\frac{1}{3}} f^{-\frac{2}{3}} & f<f_{\text {trans }} \\
0.014 D^{-1 / 3} & v_{\frac{14}{3}} f^{-\frac{17}{3}} \quad \text { otherwise }
\end{array}\right. \\
& f_{\text {trans }}=0.445 v_{-\frac{1}{3}}^{-\frac{1}{5}} v_{\frac{14}{3}}^{\frac{1}{5}}
\end{aligned}
$$

Where $D$ is the telescope aperture and the moment $v_{n}$ is defined by $v_{n}=\int_{0}^{\infty} v^{n}(z) C_{n}^{2}(z) d z$, where $v(z)$ and $C_{n}^{2}(z)$ are the turbulent velocities and refractive-index structure parameter, respectively.

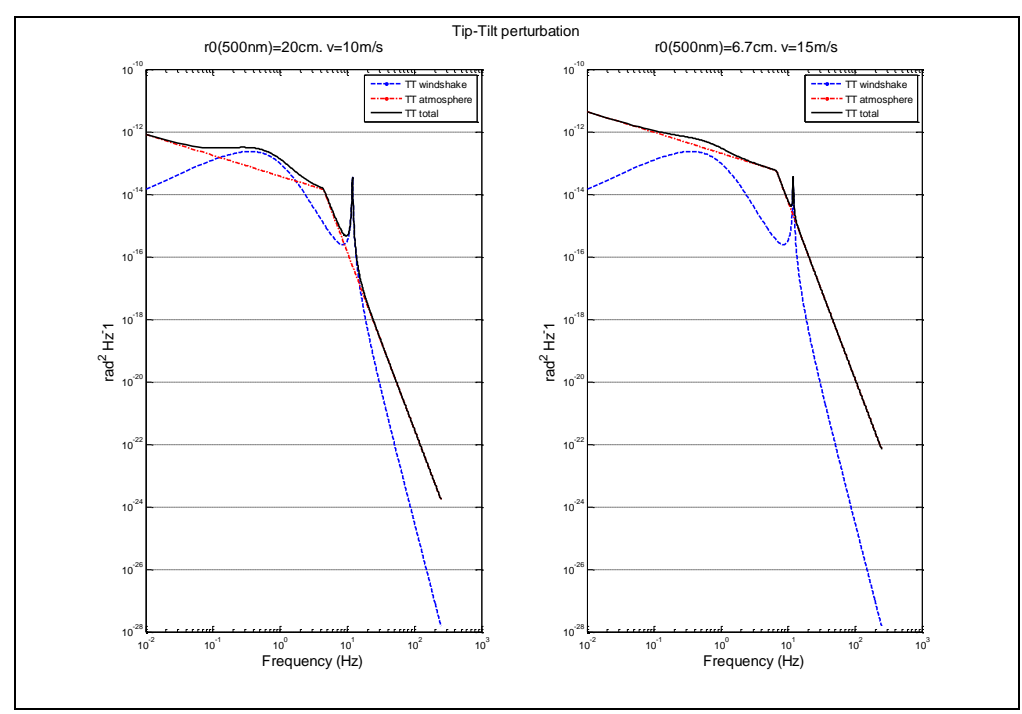

Figure 3. Tip-tilt wavefront aberration for two different atmospheres. GTCAO standard seeing scenario (left), adverse atmosphere (right).

\subsection{Spatial High order}

In this document it is called spatial high order modes to all modes except piston and tip-tilt and the expression used for the power spectrum of such modes has been extracted from [8]:

$$
\begin{array}{rlr}
p s(f)= & 0.132\left(\frac{2 \pi}{\lambda}\right)^{2} D^{4} \mu_{0}^{\frac{12}{5}} v_{\frac{5}{3}}^{-\frac{7}{5}} f^{\frac{4}{3}} & f<f_{\text {trans }} \\
0.0326\left(\frac{2 \pi}{\lambda}\right)^{2} v_{\frac{5}{3}} f^{-\frac{8}{3}} & \text { otherwise } \\
f_{\text {trans }}= & 0.705 D^{-1} \mu_{0}^{-\frac{3}{5}} v_{\frac{5}{3}}^{\frac{3}{5}} &
\end{array}
$$

Where $D$ is the telescope aperture, $\lambda$ is the correction wavelength, and the moments $\nu_{n}$ and $\mu_{n}$ are defined by $\mu_{n}=\int_{0}^{\infty} z^{n} C_{n}^{2}(z) d z$ and $v_{n}=\int_{0}^{\infty} v^{n}(z) C_{n}^{2}(z) d z$, where $v(z)$ and $C_{n}^{2}(z)$ are the turbulent velocities and refractive-index structure parameter, respectively. These functions have been estimated assuming the single-layer 'standard seeing scenario'. 


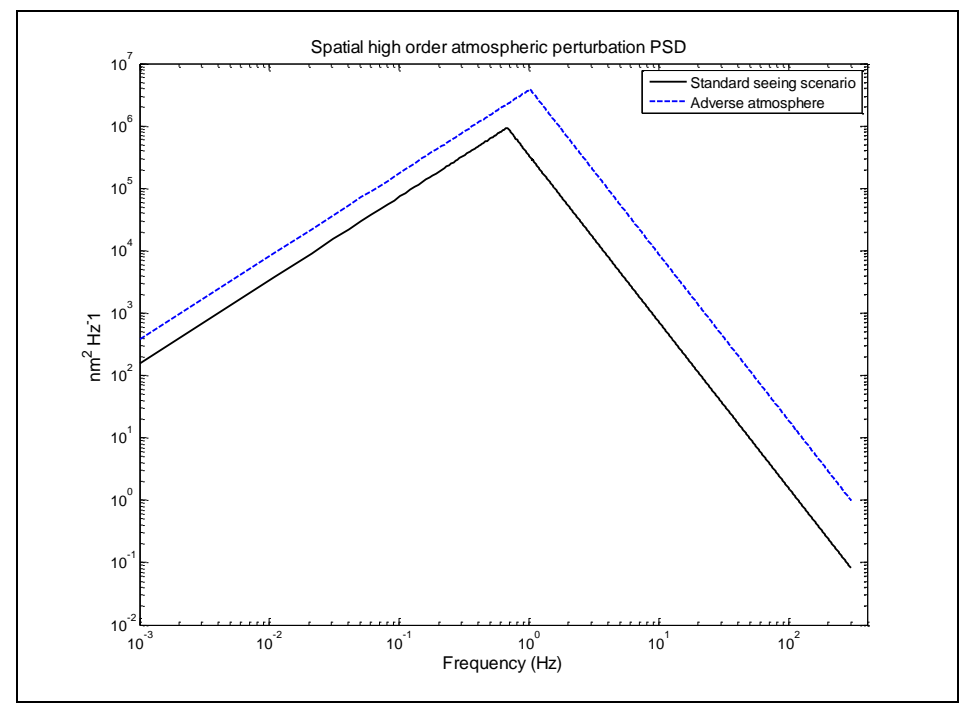

Figure 4. Spatial high order perturbation for two different atmospheres: GTCAO standard seeing scenario (continuous line), adverse atmosphere (dashed line).

\section{BANDWIDTH ERROR}

GTCAO system error Budget includes 17 elements but in this paper we will pay attention only to the bandwidth error for tip-tilt and high order modes, because they are the ones affected by the servo control and the disturbance PSD. Two models have been created using Matlab: one for tip-tilt modes and one for high order optical modes.

\subsection{TIP-TILT}

The low order model in Figure 5, described in the $\mathrm{z}$ transform, shows that the tip-tilt measured by the WFS is used as input for the DM controller and also for the M2 controller. There is an additional vertical connection between the CCDM output and the LPF_M2 which functionality is to off-load the DM from the accumulated tip-tilt actuation. That tiptilt actuation in the DM will correct mostly the disturbances produced by the atmospheric, windshake and telescope but also very slow drift in the hardware itself.

One advantage of this scheme over other ones, that has been discarded and will be mentioned later, is that M2 and the DM can be used simultaneously or actuating only on one of them. This is useful at the beginning of the AO operation where M2 can start correcting slow tip-tilt to help locating the guide star in the WFS sub-apertures and a few seconds later the DM starts to correct simultaneously with M2. This can also be used for debugging or maintenance. In regular closed loop operation both mirrors are correcting at the same time and the role of M2 is mostly off-loading the DM because the tip-tilt measured by the WFS will be corrected by the DM due to its faster dynamics compared to M2.

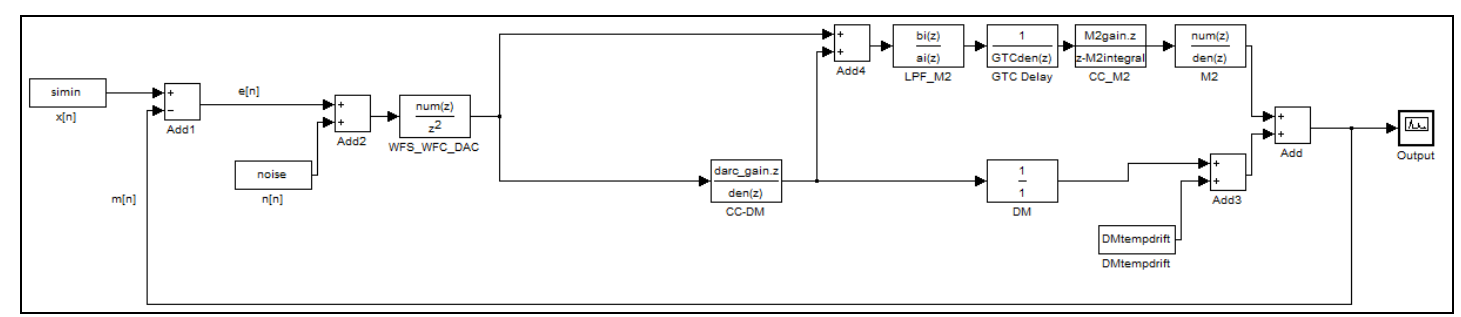

Figure 5. Block diagram for tip-tilt modes.

The model includes, hence, 2 controllers, one for the DM and one for M2. A preliminary tunning of both, fulfilling with a phase margin $>30^{\circ}$ and a gain margin $>6 \mathrm{~dB}$, produces the following error bode plot, Figure 6 , which analytical definition is: 


$$
E(z)=\frac{1}{1+G(z)}
$$

Where G(z) is the open loop transfer functiom and where the standard seeing scenario for a guide start magnitude of 11 has been considered, using the optimum exposure time which is $2 \mathrm{~ms}$ for that case. In such conditions the bode plot of the $\mathrm{E}(\mathrm{f})$ function shows that bandwidth is near $40 \mathrm{~Hz}$ and the sensibility peak is below $6 \mathrm{~dB}$, producing a wavefront residual bandwidth error after correction of $42 \mathrm{~nm}$.

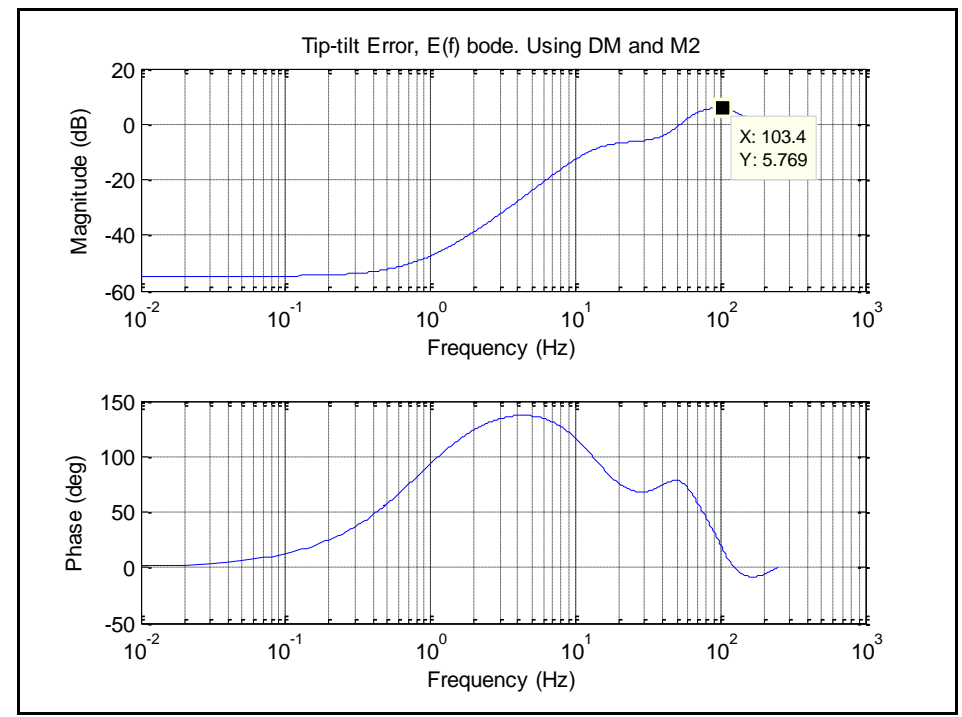

Figure 6. Tip-tilt error function.

There are other two tip-tilt schemes that have been discarded. The first one, in Figure 7, includes an additional high pass filter previous to the DM controller, so that the slow tip-tilt measured by the WFS is only corrected by M2 but not by the DM. It was discarded because the HPF introduces certain delay to the DM loop that may become important at high frequencies. Additionally the slow DM thermal drifts in the DM hardware would not be off-loaded by M2 because the M2 compensation would not reach the DM in the following cycles due to the HPF, hence the WFS and the DM tip-tilt drift would be contradictory inputs for the M2 loop.

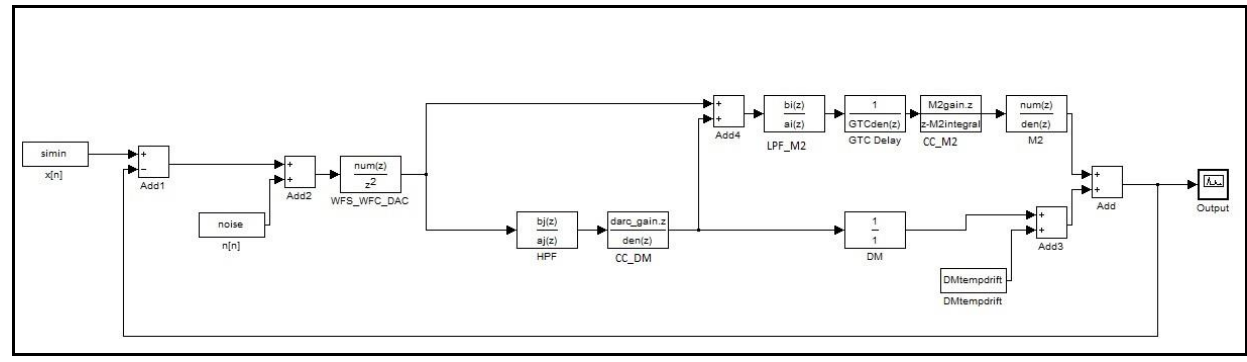

Figure 7. Tip tilt discarded alternative design 1. Includes a high pass filter in the DM branch.

The second discarded scheme, in Figure 8, is quite similar to the one in Figure 5, but removing the direct connection between the WFS and the M2 LPF. Both of them achieve very similar residual errors. The drawback of Figure 8 is that it cannot use M2 without DM because it becomes unstable, unless CC_DM controller is bypassed when the DM is not active. This additional bypass implementation is not required in Figure 5 and therefore scheme in Figure 8 has been discarded. 


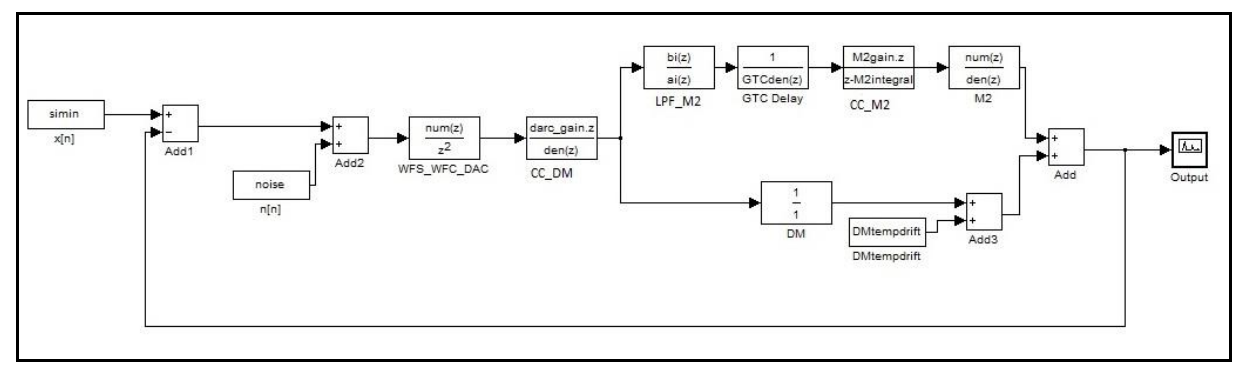

Figure 8. Tip-tilt discarded alternative design 2. There is not direct connection between WFS and M2 Loop.

\subsection{HIGH ORDER MODES}

The high order optical modes, all except piston, tip and tilt, have been modelled using the following scheme in the $\mathrm{z}$ transform.

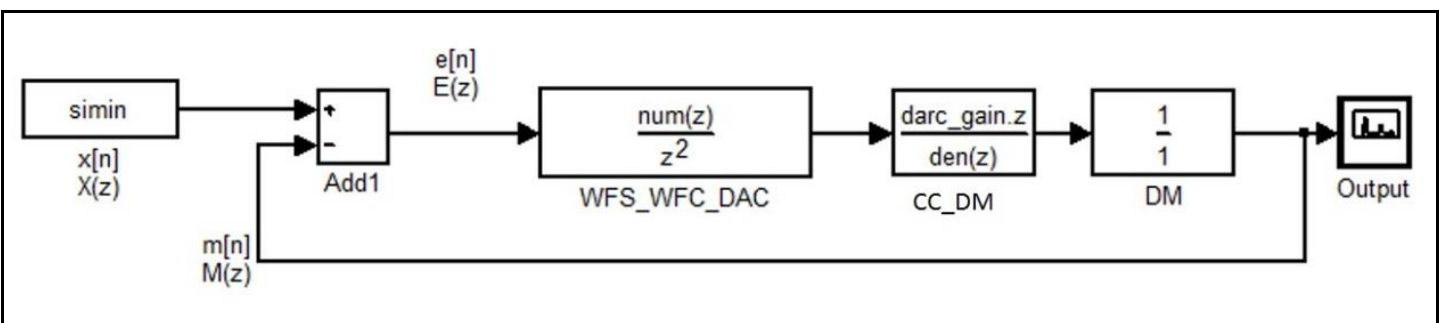

Figure 9. Block diagram used to calculate the bandwidth error contribution due to high order optical modes.

In the controller block CC_DM, 2 different controllers have been tried: a leak Proportional Integral (PI) as it is implemented by default in the Durham Adaptive Optics Real time Controller (DARC)[9][10] platform, eq. 4, and a double integrator as in eq. 5 .

$$
\begin{aligned}
& C C_{D M}(z)=\frac{\text { darc gain }}{1-\text { darc }_{\text {decayfactor }} z^{-1}} \\
& C C_{D M}=K_{1}\left[\frac{1}{1-z^{-1}}+k_{2} \frac{1}{\left(1-z^{-1}\right)^{2}}\right]
\end{aligned}
$$

When both of them are tuned correctly, a very similar wavefront residual error is achieved but the frequencies where they reject better the disturbances are not the same. Due to the second pole, the double integrator has a higher attenuation at very low frequencies. The implementation proposed for GTCAO in the following section allows both controllers and the choice depends on the disturbance PSD to reject which at present is the model described previously but will be updated by real measurements. The bode plot of the error function, Figure 10, shows both controller structures tuned to fulfil the phase margin $>30^{\circ}$ and a gain margin $>6 \mathrm{~dB}$. That error function in combination with the high order modes disturbance for the standard seeing scenario produces a wavefront residual bandwidth error after correction of $32 \mathrm{~nm}$. 


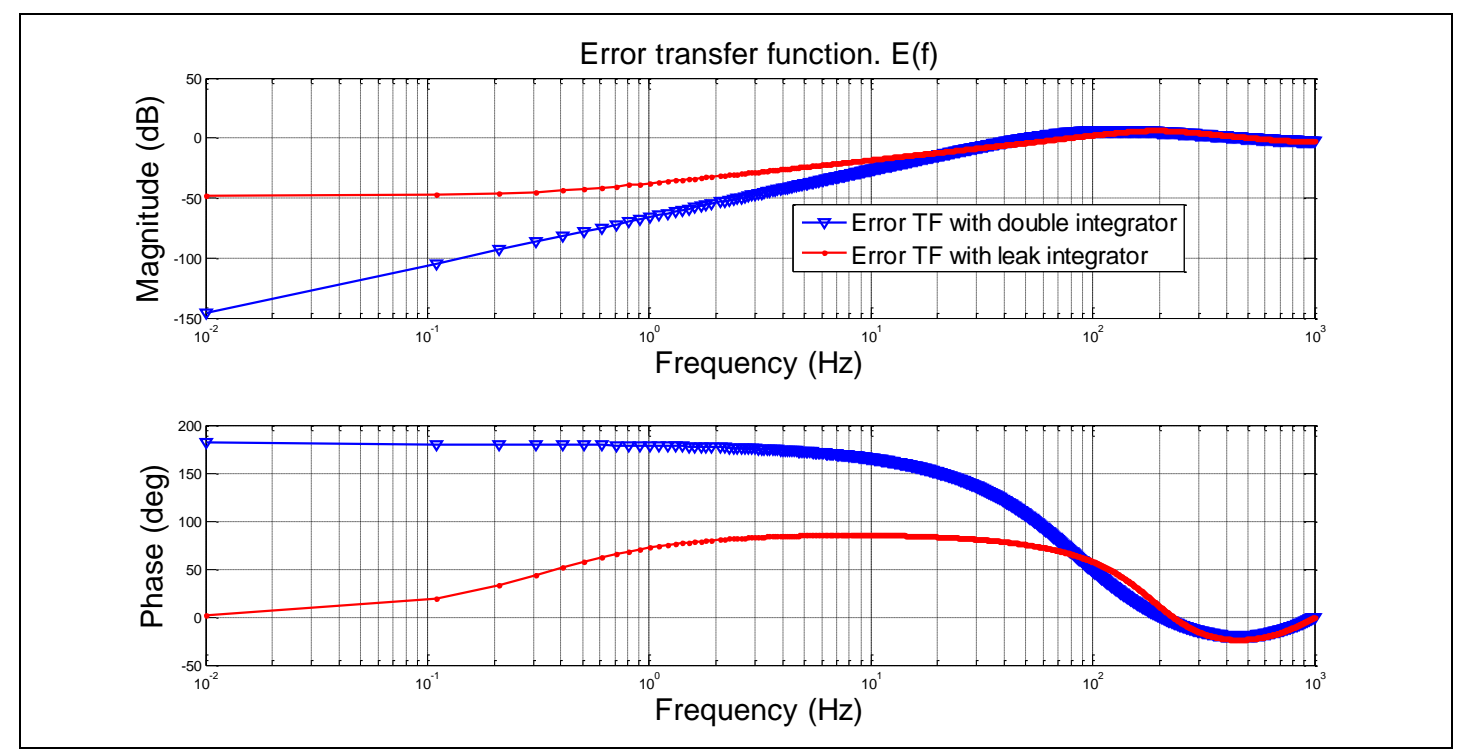

Figure 10. Bode of the Error transfer function in Eq. 8. For two different controller configuration: with leak integrator (red with spots) and with double integrator (blue with triangles).

\section{GLOBAL SCHEME AND FUTURE WORK}

Putting tip-tilt and high order modes all together the global scheme is created in Figure 11. It includes elements to test the discarded tip-tilt schemes, in case the decisions are reviewed in the laboratory. It is also added a branch to off-load DM with M1 based on WFS measurements and actuation in the DM. The DM commands include 110 elements: global focus, global coma and $36^{*}$ (piston+tip+tilt). The tip-tilt modes have been projected out of the high order (HO) line, to avoid summing them twice in the DM.

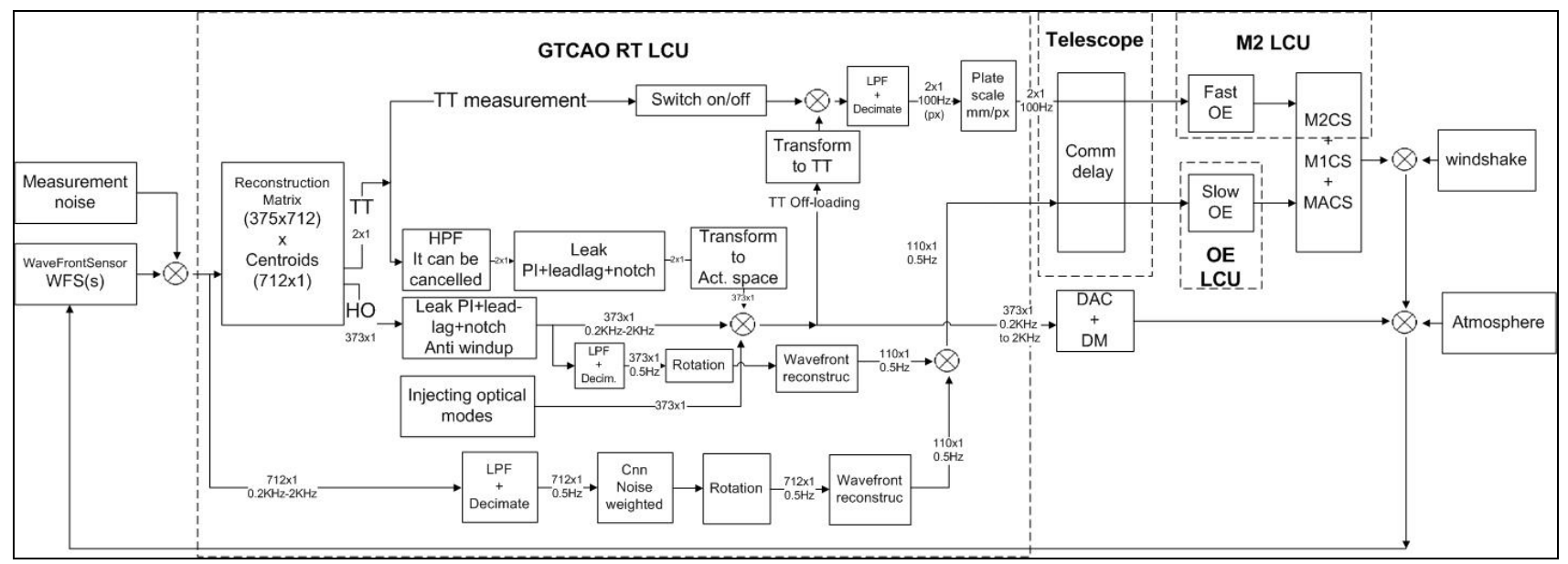

Figure 11. General servo control block diagram in GTCAO.

Concerning the future work, the scheme in Figure 11 is being implemented into the DARC[9][10] platform and is being integrated with the Durham Adaptive Optics Simulation Platform (DASP) for simulating the wavefront residual error after correction while running the scheme in Figure 11, which is the same controller that is proposed to be used in the laboratory and later in the telescope. Simultaneously the integration and verification of the opto-mechanical elements in the GTCAO optical bench is being carried out. The present scheme will allow testing the DM loop and M2 loop separately, which is important because there will be no tip-tilt mirror in the laboratory but only in the telescope. 
In a second stage of servo control development, different strategies may be tested based on an modal approach, where disturbance is decomposed in Zernike modes or Karhunen Loeve modes, having each mode its own controller tuning.

\section{ACKNOWLEDGEMENTS}

This activity is funded by the Canary Islands Local Government, within the program "Canarias objetivo de progreso" promoted by the European Regional Development Fund of the European Union, operative program 2014-2020.

It is pre-financed through a loan from the Spanish Ministry of Economy (State Secretary for Research).

\section{REFERENCES}

[1] Bello, D., Boucher, L., Rosado, M., Castro López, J., Feautrier, P., "Characterization of the main components of the GTCAO system: 373 actuators DM and Ocam2 camera”, Proc. Third AO4ELT Conference, vol. 1 (2013).

[2] Bello, N. et at, "Characterization of the main components of the GTCAO system", 3rd AO4ELT

[3] Devaney, N. "Preliminary design and plans for the GTC adaptive optics system", SPIE 5490 P913

[4] Femenía, B. \& Devaney, N., 2003, A\&A, 404, 1165

[5] Lopez, J.A. et ak, "FRIDA: Integral-field spectrograph and imager for the adaptive optics system of the Gran Telescopio Canarias", Proceedings 62693R SPIE , (2006)

[6] B. García-Lorenzo, A. Eff-Darwich, J.J. Fuensalida and J. Castro-Almazán (2009). "Adaptive optics parameters connection to wind speed at the Teide Observatory". Monthly Notices of the Royal Astronomical Society 397

[7] Glenn A. Tyler. Bandwidth considerations for tracking through turbulence. 1994

[8] "Laser-guide-star systems for astronomical applications", R.R. Parenti \& R.J. Sasiela, J. Opt. Soc.Am. A, 11, 288-308 (1994)

[9] Basden A, Geng D, Myers R, Younger E., "Durham Adaptative optics real time controller", Applied Optics. 2010 Nov 10;49(32):6354-63. doi: 10.1364/AO.49.006354.

[10] Basden, A. G. (2014). "A real-time simulation facility for astronomical adaptive optics", Monthly Notices of the Royal Astronomical Society 439(3): 2854-2862. 